\title{
ELECTRIC FIELD STIMULATED EMISSION OF ELECTRONS FROM DEEP TRAPS IN $\mathrm{SiO}_{2}$
}

\author{
I. Strzalkowski, M. Marczewski, M. Kowalski and C. Jastrzębski
}

Institute of Physics, Warsaw University of Technology

Koszykowa 75, 00-662 Warszawa, Poland

\begin{abstract}
An electric field induced electron tunneling emission from deep traps and an energy distribution of trap levels in VLSI grade $\mathrm{SiO}_{2}$ layers have been studied using a new isochronal - EFSE - technique. A broad spectrum with a density of trap states peak at about $1.9 \mathrm{eV}$ was observed for the first time. The experiment proved the importance of an electron trap-to-band tunneling emission in $\mathrm{SiO}_{2}$
\end{abstract}

PACS numbers: 72.20.Jv, 73.40.Qv, 73.60 Hy

\section{Introduction}

Electron trapping and detrapping due to intrinsic and generated defects in $\mathrm{SiO}_{2}$ layers employed as the insulator in MOSFETs or EEPROMs are still of primary importance. During device operation, electric field in $\mathrm{SiO}_{2}$ induces simultaneously a number of different electron and hole transitions [1], among which a significant one is related to emission of electrons from traps due to trap-to-band tunneling. The occurence of this process in electron trapping, detrapping and trap generation experiments makes difficult a determination of the real values of trap parameters and trapping, detrapping, generation coefficients. Thus, the aim of the work was to study the effect of electric field induced electron tunneling emission from oxide trap centers and to use this effect for determination of trap energy levels.

\section{Experimental details}

Investigated $\mathrm{SiO}_{2}$ layer was integrated-circuit grade, amorphous, dry, thermally grown oxide with thickness of $490 \AA$. It was incorporated as the gate oxide into an $n$-channel Si MOSFET-type test structure. To study an electron trap-to-band tunneling emission and measure an energy distribution of oxide traps we used a new experimental technique, named isochronal-electric field stimulated emission (I-EFSE) technique [2].

I-EFSE technique consists of the following four steps: (1) the electron traps are filled by capturing electrons injected into the oxide, (2) the electron injection 
into the oxide is stopped, (3) the oxide electric field applied for stimulation of tunnel emission of trapped electrons to the oxide conduction band is increased in increments and maintained constant for the same isochronal time $\tau$ at each field increment, and (4) at the end of each step the trapped electron density is measured to monitor the electron detrapping.

The experiments were carried out at room temperature, so all shallow traps had been emptied due to thermal electron emission, leaving electrically active only deep oxide traps. Trap filling was performed by nonavalanche injection of hot electrons into $\mathrm{SiO}_{2}$ conduction band [3] with the fluence of $2.5 \times 10^{18} \mathrm{e} / \mathrm{cm}^{2}$ (sufficient for charging of deep traps with small capture cross sections: $10^{-18}-10^{-19} \mathrm{~cm}^{2}$ ) at low oxide electric field, $1 \mathrm{MV} / \mathrm{cm}$, and injection current density $2.0 \mu \mathrm{A} / \mathrm{cm}^{2}$, in order to avoid the generation of new traps. The oxide electric field during electron tunnel emission was increased in $0.1 \mathrm{MV} / \mathrm{cm}$ steps and maintained constant for $\tau=300 \mathrm{~s}$ at each step. At the end of each step the drain-source current versus gate-source voltage, $I_{\mathrm{ds}}\left(U_{\mathrm{sg}}\right)$, characteristic was measured, and from the voltage shift of this characteristic the density of trapped charge $Q_{t}$ remaining in oxide traps after each electron emission step via tunneling was determined [3]. The assumed value of the trapped charge centroid was a half of the oxide thickness, which corresponds to the uniform trapping model.

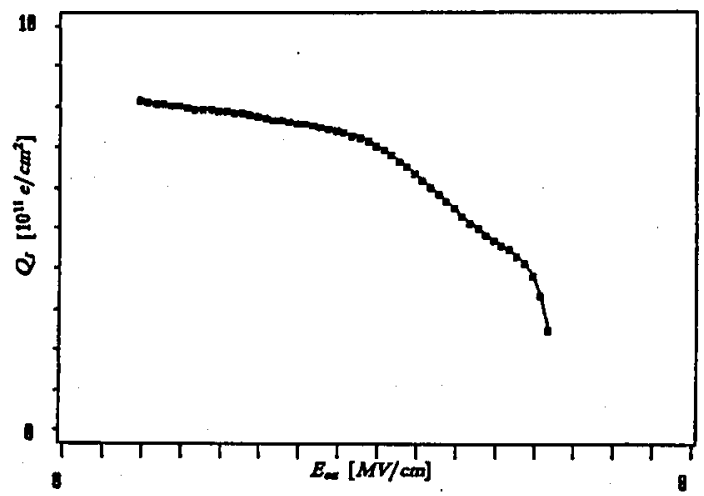

Fig. 1. The density of trapped charge $Q_{t}$ remaining in the oxide after each emission step versus discharge electric oxide field $E_{\text {ox }}$.

Figure 1 shows the density of trapped charge $Q_{t}$ remaining in the oxide after each emission step versus discharge electric oxide field $E_{\text {ox }}$. The curve has a maximum slope at a discharge oxide field of approximately $5.0 \mathrm{MV} / \mathrm{cm}$, which occurs when the largest number of electrons are detrapped in a unit energy range. The energy spectrum of oxide traps was calculated from the experimental emission decay data according to the tunneling emission model described in the next part. 


\section{Results and discussion}

The areal density of electrons that remain trapped after a tunnel emission time $\tau$ at an oxide field $E_{\text {ox }}$ is

$$
n_{t}=\int_{E_{\mathrm{v}}}^{E_{c}} \exp (-\omega \tau) D_{t}\left(E_{t}\right) \mathrm{d} E_{t},
$$

where $D_{t}\left(E_{t}\right)$ is the density of states of charged oxide electron trap at energy $E_{t}$, measured from the oxide conduction band edge $E_{\mathrm{c}}, E_{v}$ is the energy of the oxide valence band edge, and $\omega$ is the tunneling emission rate. In the CCK (Chaudhuri, Coon and Karunasiri) tunneling emission model [4], derived for general "quantum defect"-to-band tunneling the transition rate is given by

$$
\omega=\omega_{0}\left(\alpha / E_{\text {ox }}\right)^{2 n-1} \exp \left(-\alpha / E_{\text {ox }}\right)
$$

where

$$
\alpha=\frac{4\left(2 m^{*}\right)^{1 / 2} E_{t}^{3 / 2}}{3 e h}, \quad \omega_{0}=\frac{6^{2 n} E_{t}|S(n)|^{2}}{3 h \Gamma^{2}(n+1)},
$$

and

$$
S(n)=\frac{\pi}{\sin (n \pi)}\left[\frac{1}{2} \sum_{m=0}^{\infty} \frac{1}{(n-m-1)^{2}(n-m)^{2}}\right]^{-\frac{1}{2}} .
$$

The parameter $n$ depends on the nature of the trap binding potential, i.e. whether a trap is shallow, deep, charged or neutral [4]. For deep and neutral traps, i.e. likely our case, $n$ is close to zero and $S(n)=\sqrt{2}$ (for shallow Coulombic centers, $n$ is close to one and $S(n)=1$ ).

The areal density of charged oxide traps after $i$-th field increment is

$$
n_{\mathrm{ti}}\left(E_{\text {oxi }}\right)=\int_{E_{\mathrm{v}}}^{E_{\mathrm{c}}} D_{t}\left(E_{t}\right)\left[\exp (-\omega \tau) \exp \left(-\omega_{i-1} \tau\right) \cdot \ldots \cdot \exp \left(-\omega_{1} \tau\right)\right] \mathrm{d} E_{t} .
$$

Since $\exp \left(-\omega_{i} \tau\right)$ is a double exponential function in $E_{\mathrm{ti}}$ it can be approximated by a step function in energy, so experimentally determined value of $n_{t i}\left(E_{\text {oxi }}\right)-$ $n_{\mathrm{ti}+1}\left(E_{\mathrm{ox}+1}\right)$, where $N_{\mathrm{ti}}=Q_{\mathrm{ti}} / e$, can be expressed as

$$
n_{\mathrm{ti}}-n_{\mathrm{ti}+1} \approx \int_{E_{\mathrm{ti}}}^{E_{\mathrm{ti}+1}} D_{t}\left(E_{t}\right) \mathrm{d} E_{t} \approx D_{t}\left(E_{\mathrm{ti}}\right)\left(E_{\mathrm{ti}+1}-E_{\mathrm{ti}}\right) .
$$

$E_{\mathrm{ti}}$ for a given $E_{o x i}$ is calculated from the solution of the equation $\partial^{2} \exp (-\omega \tau) / \partial E_{t}^{2}=0$, thus it is dependent on the tunneling emission model used. Then the density of states was calculated from the expression:

$$
D_{t}\left(E_{\mathrm{ti}}\right) \approx\left[n_{\mathrm{ti}}\left(E_{\mathrm{oxi}}\right)-n_{\mathrm{ti}+1}\left(E_{\mathrm{oxi}+1}\right)\right] /\left(E_{\mathrm{ti}+1}-E_{\mathrm{ti}}\right),
$$

using the following assumptions: $n=0$ (deep, neutral traps), $m^{*}=m_{0}$ (the free electron mass) [2]. The energy spectrum for the existing discharged oxide traps, $D_{t}$ vs. $E_{t}$ is shown in Fig. 2. One can see a nearly Gaussian-shape distribution of traps peaked at $1.93 \mathrm{eV}$ with a half-width of $0.54 \mathrm{eV}$ and maximum density of states $6.81 \times 10^{11} \mathrm{~cm}^{-2}$. The broadness of the density spectra is characteristic of amorphous materials. The obtained energy level at the peak density is deeper 


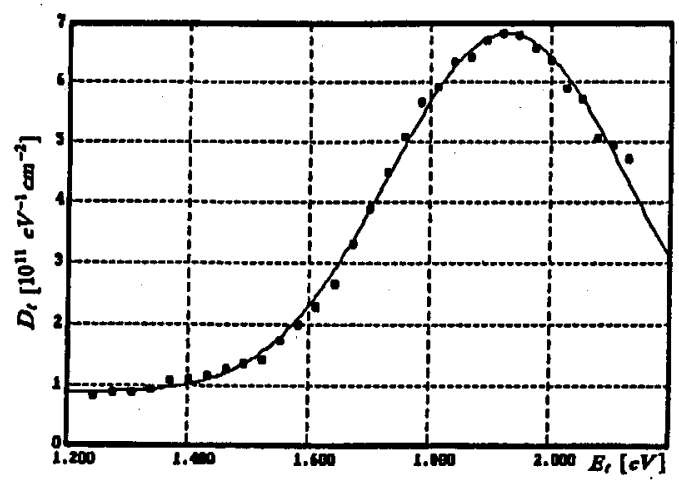

Fig. 2. The density of states of discharged oxide traps $D_{t}$ versus trap energy $E_{t}$. Solid line represents Gaussian curve fitted to the experimentally determined points.

than theoretically predicted (1.0-1.4 eV) acceptor-type level for oxygen vacancy center, the most typical point defect in amorphous $\mathrm{SiO}_{2}$.

It should be pointed out that obtained results depend on the assumptions that the only electric field stimulated emission process is the tunneling transition of electrons from trap-to-band and that this process is well described by the CCK model. The parameters, like location of trapped charge centroid, altering the oxide field by trapped charge, the energy dependence of the capture cross section, the value of effective electron mass used for calculation can also be important, though in the light of the paper [2] calculated energy spectrum of traps is not strongly affected by these parameters.

Discharging of oxide layer by electric field via tunneling emission could be practically important, offering possibility of "cleaning" of device oxide layers charged during long lasting operation.

\section{References}

[1] C.T. Sah, Fundamentals of Solid State Electronics, World Scientific, Singapore 1992.

[2] E. Thompson, T. Nishida, J. Appl. Phys. 70, 6864 (1991).

[3] I. Strzałkowski, M. Marczewski, M. Kowalski, J. Wisłowski, Appl. Phys. A 51, 19 (1990).

[4] S. Chaudhuri, D.D. Coon, R.P.G. Karunasiri, J. Appl. Phys. 54, 5476 (1983); S. Chaudhuri, D.D. Coon, G.E. Derkits, J.R. Banavart, Phys. Rev. A 23, 1657 (1981). 